Supporting Information for

\title{
High Efficiency Tandem Thin-Perovskite/Polymer Solar Cells with a Graded Recombination Layer
}

Yao Liu, ${ }^{\dagger}$ Lawrence A. Renna, ${ }^{\star}$ Monojit Bag, ${ }^{\ddagger}, \$$ Zachariah A. Page ${ }^{\dagger}$ Paul Kim, ${ }^{\dagger}$ Jaewon Choi, ${ }^{\dagger}$ Todd Emrick, ${ }^{\dagger} *$ Dhandapani Venkataraman, ${ }^{*} *$ and Thomas P. Russell ${ }^{\dagger, *}$

${ }^{\dagger}$ Department of Polymer Science \& Engineering, Conte Center for Polymer Research 120 Governors Drive, University of Massachusetts, Amherst, MA 01003, USA

$\$$ Department of Chemistry, 710 North Pleasant Street, University of Massachusetts, Amherst, MA 01003-9303, USA

${ }^{\$}$ Department of Physics, Roorkee 247667, Indian Institute of Technology, Roorkee, Uttarakhand, India

\section{Corresponding Author}

*E-mail: russell@mail.pse.umass.edu (T.P. R.);dv@chem.umass.edu; tsemrick@mail.pse.umass.edu 
Materials: $\mathrm{C}_{60}-\mathrm{N}$ and $\mathrm{C}_{60}-\mathrm{SB}$ are prepared according to previous procedures. ${ }^{46} \mathrm{PCE}-10$ and MAI was purchased from 1-Material. Both $\mathrm{PC}_{71} \mathrm{BM}$ and $\mathrm{PC}_{61} \mathrm{BM}$ were purchased from Nano-C. All the solvents and Lead acetate used in this research were purchased from Sigma Aldrich and used without further purification.

KPFM: The $\mathrm{V}_{\mathrm{CPD}}$ is measured by the $\mathrm{DC}$ bias applied via a feedback loop to the AC oscillating probe to null any mechanical oscillations caused by the probe/sample potential differences, thus $\mathrm{V}_{\mathrm{CPD}}=\mathrm{V}_{\mathrm{DC}}{ }^{48}$ When performing KPFM experiments, we grounded the samples in order to prevent the buildup of excess charges during raster scan. All the measurements were performed in air.

UV-visible: The absorptions of perovskite film and polymer film on glass/PEDOT:PSS substrates were measured on Shimadzu UV 3600. Attenuation coefficients were determined by casting three relatively thick films (polymer BHJ: $\sim 160 \mathrm{~nm}$; perovskite film: $\sim 330 \mathrm{~nm}$ ) onto glass/PEDOT:PSS substrates, measuring their absorption profiles with UV-Vis absorption spectroscopy, determining thickness using profilometry and taking the average values from both measurements as A (absorption, $\mathrm{AU}$ ) and 1 (path length, $\mathrm{cm}$ ) to determine the attenuation coefficient $\left(\alpha, \mathrm{cm}^{-1}\right)$ using the Beer-Lambert law for films: $\alpha=\mathrm{A} / 1$.

PXRD: Powder X-Ray diffraction was performed on a PANalytic X'Pert ${ }^{3}$ X-Ray diffractometer with a Ni filter, $1 / 2$ " diverging slit, vertical goniometer, and $\mathrm{X}^{\prime}$ Celerator detector. Measurements were made from $2 \theta=5^{\circ}-60^{\circ}$ under $\mathrm{Cu} \mathrm{K-Alpha}(1.542 \AA)$.

AFM: Atomic force microscopy was performed on a Digital Instruments Dimension 3100, operating in tapping mode.

SEM: Scanning electron microscopy (SEM) and cross-sectional SEM was performed on a FEI Magellan 400 FESEM. 


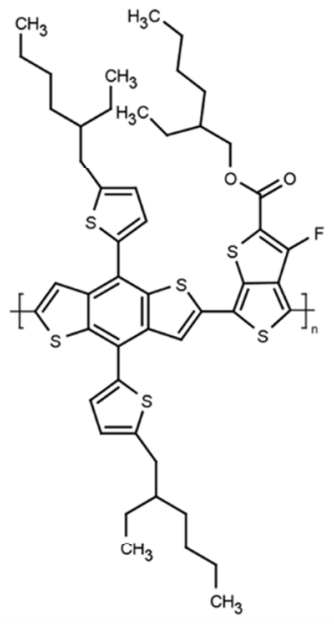

PCE-10

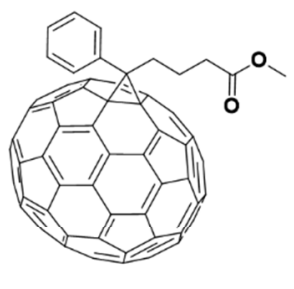

$\mathbf{P C}_{71} \mathbf{B M}$

Figure S1. Molecular structure of PCE-10 and $\mathrm{PC}_{71} \mathrm{BM}$.
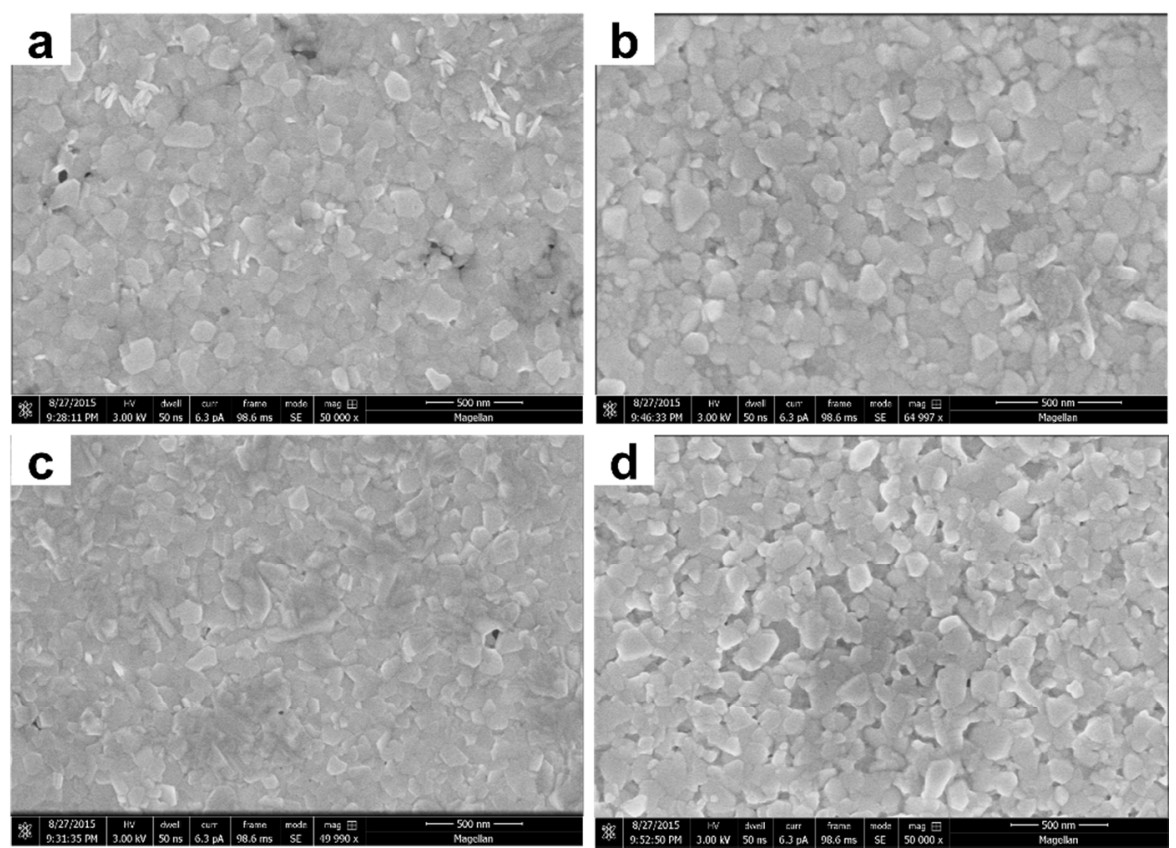

Figure S2. SEM images of perovskite films on ITO/PEDOT:PSS substrates with different film thickness. (a) $70 \mathrm{~nm}$. (b) $\sim 90 \mathrm{~nm}$. (c) $\sim 110 \mathrm{~nm}$. (d) $\sim 160 \mathrm{~nm}$. 

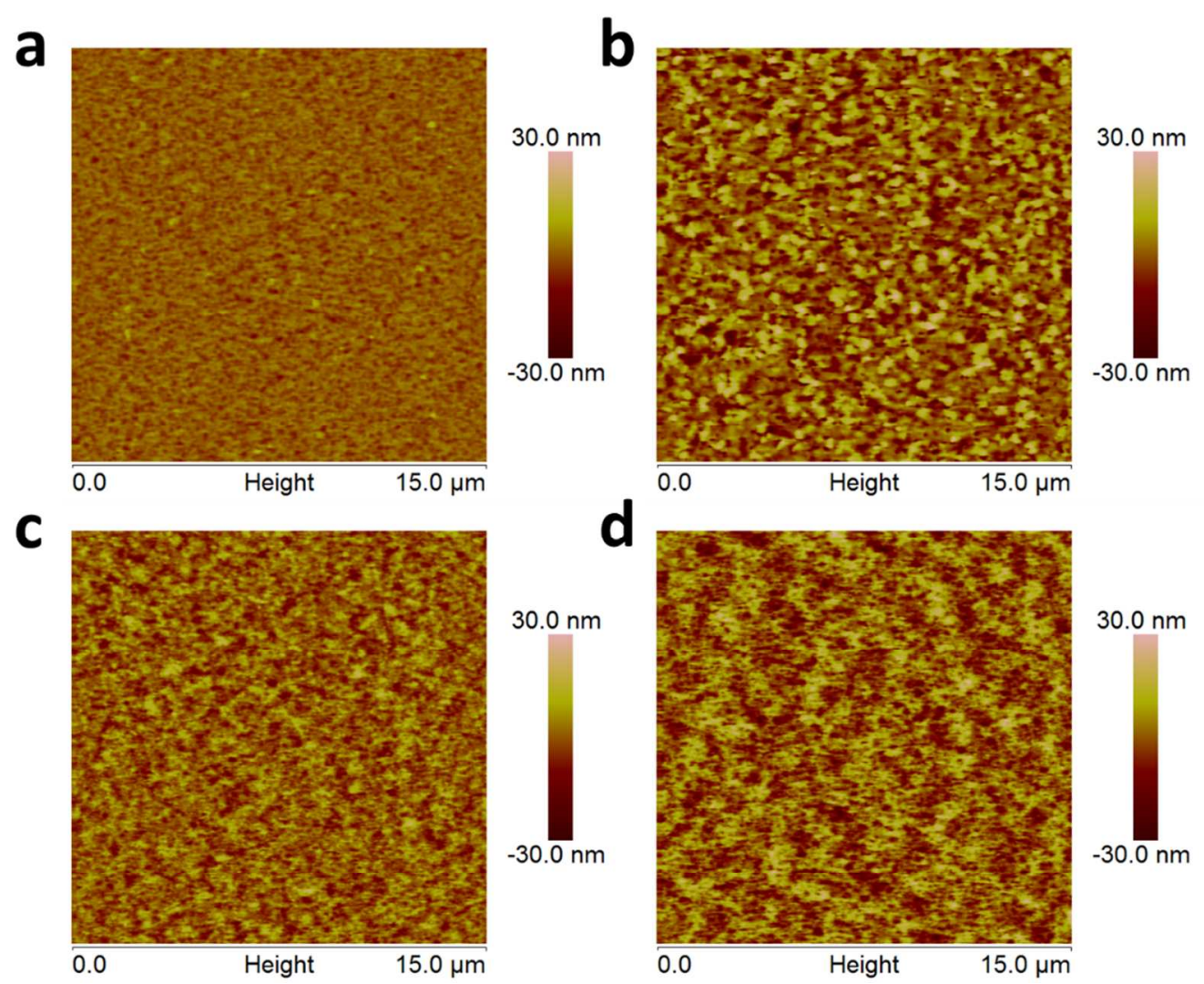

Figure S3. AFM images of perovskite films on ITO/PEDOT:PSS substrates with different film thickness. (a) $\sim 70 \mathrm{~nm}$. (b) $\sim 90 \mathrm{~nm}$. (c) $\sim 110 \mathrm{~nm}$. (d) $\sim 160 \mathrm{~nm}$.
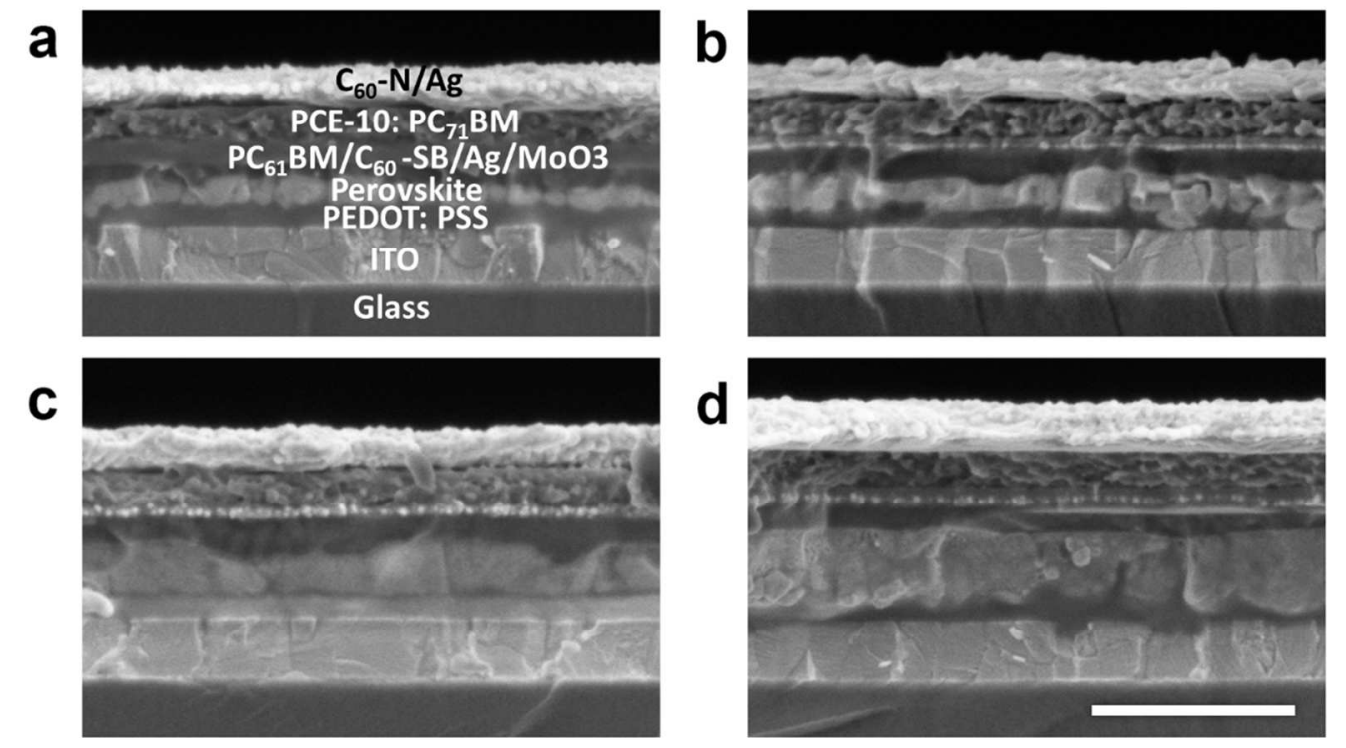

Figure S4. Cross-sectional SEM images of the tandem devices with different perovskite layer thickness. (a) $\sim 70 \mathrm{~nm}$. (b) $\sim 90 \mathrm{~nm}$. (c) $\sim 110 \mathrm{~nm}$ and (d) $\sim 160 \mathrm{~nm}$ (The scale bar is $500 \mathrm{~nm}$ ). 
a

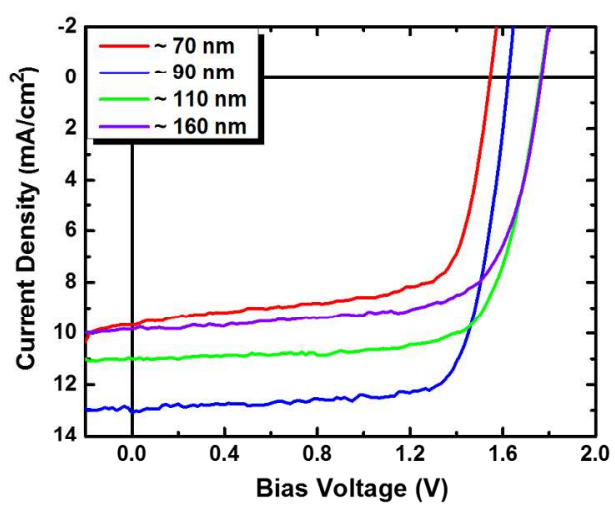

C

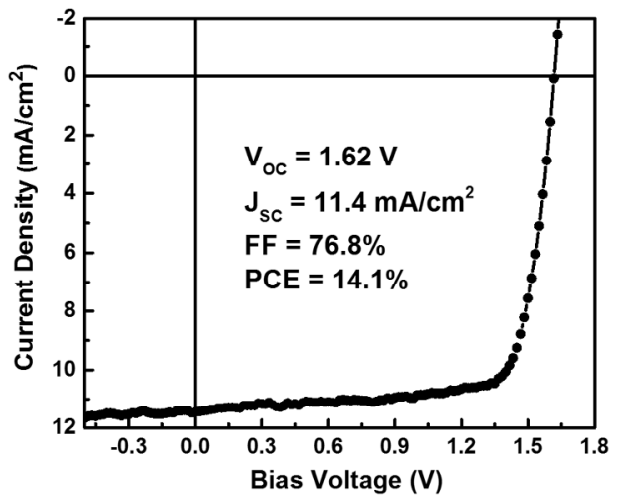

b

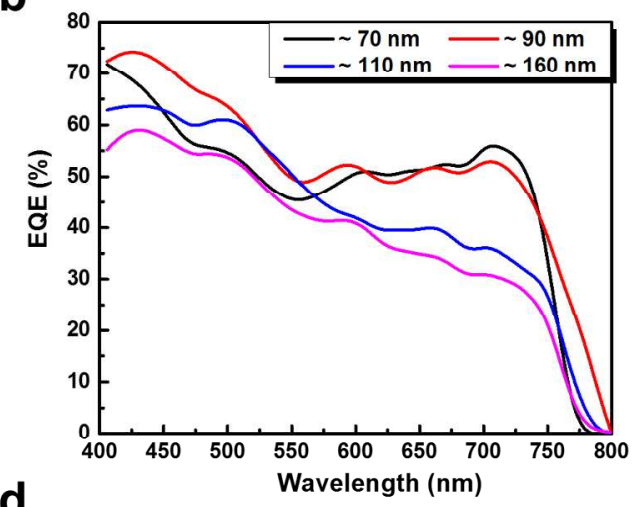

d

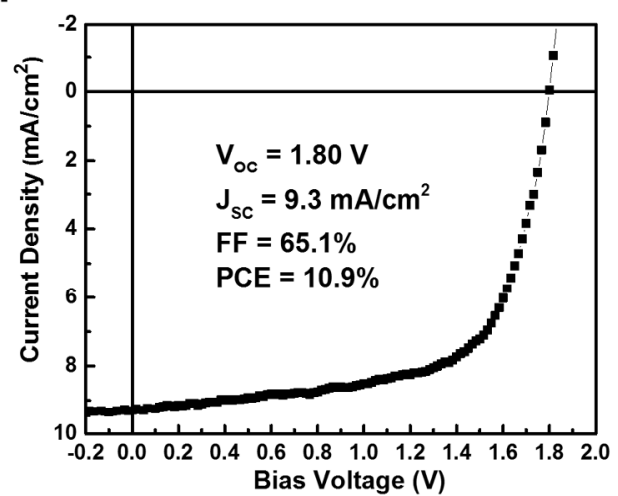

Figure S5. Device performance of perovskite/polymer tandem solar cells. (a) J-V curves of polymer/ perovskite hybrid tandem solar cells with different perovskite layer thickness. (b) EQE profiles of polymer/ perovskite hybrid tandem solar cells with different perovskite layer thickness. (c) J-V curve of polymer/ perovskite hybrid tandem solar cells with the best FF value. (d) J-V curve of polymer/ perovskite hybrid tandem solar cells with the best $\mathrm{V}_{\mathrm{OC}}$ value. 

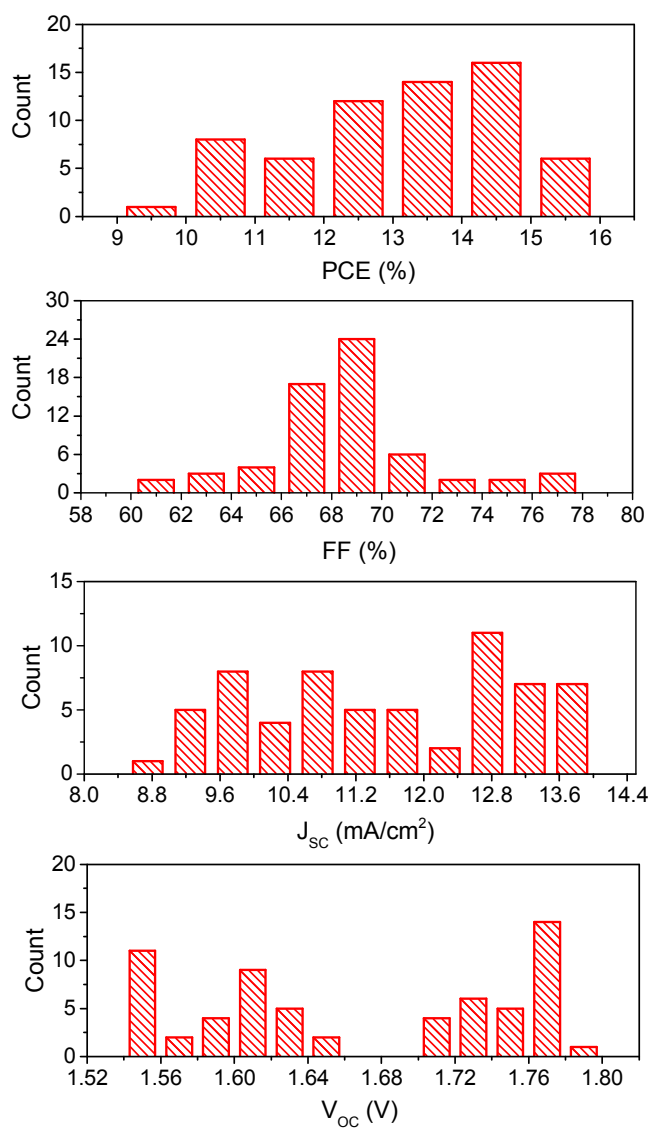

Figure S6. Histograms of PCE, FF, $\mathrm{J}_{\mathrm{SC}}$ and $\mathrm{V}_{\mathrm{OC}}$ based on 63 independent tandem devices. 


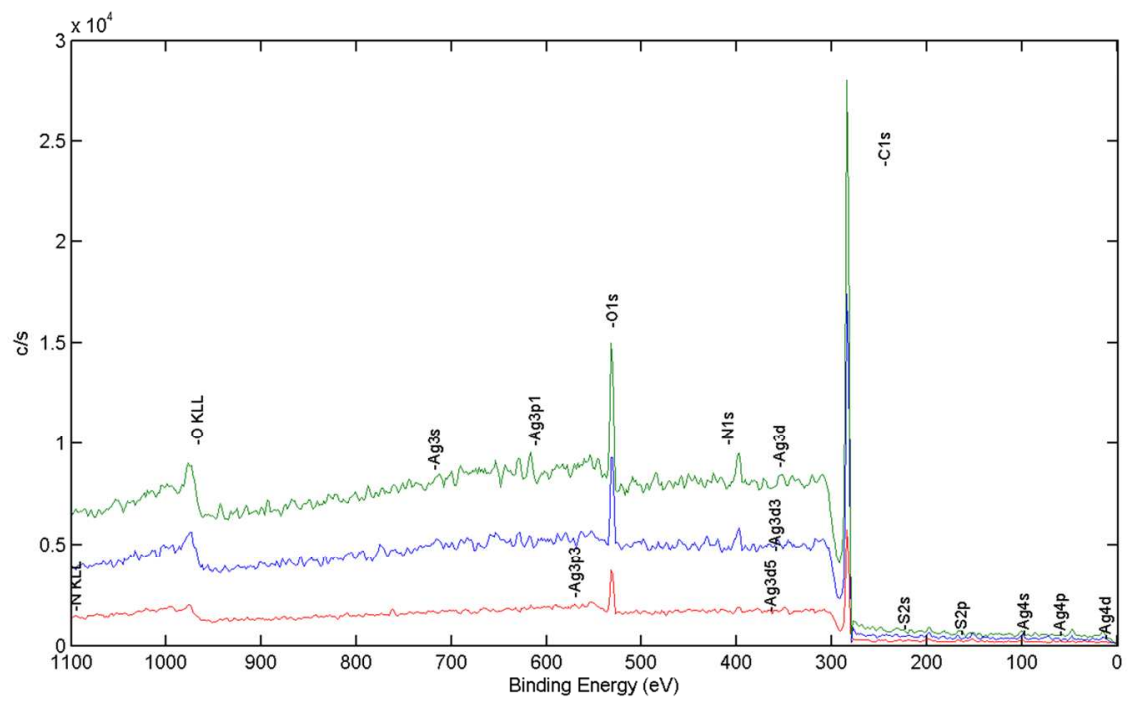

Figure S7. XPS of the Ag film surface peeled from the $\mathrm{C}_{60}$-SB layer. The angle dependent measurements show the signals of nitrogen, sulfur and oxygen coming from $\mathrm{C}_{60}-\mathrm{SB}$. Confirming its presence on the peeled Ag electrode.

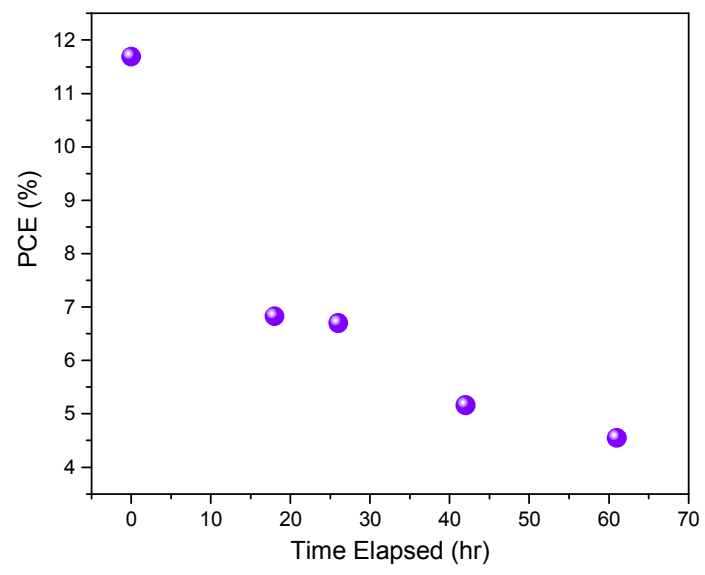

Figure S8. For the stability measurments of the tandem solar cell, we used one of our champion devices, initially show efficiency of $15.9 \%$. After storing in the glove box without encapsulation for about 9 months, the efficiency drops to $11.7 \%$. Then we took this device into air without encapsulation. The devices were stored at ambient atmosphere with a humidity of $\sim 20 \%$ and in the absence of light and the devices were brought back into the glovebox for testing each time. 


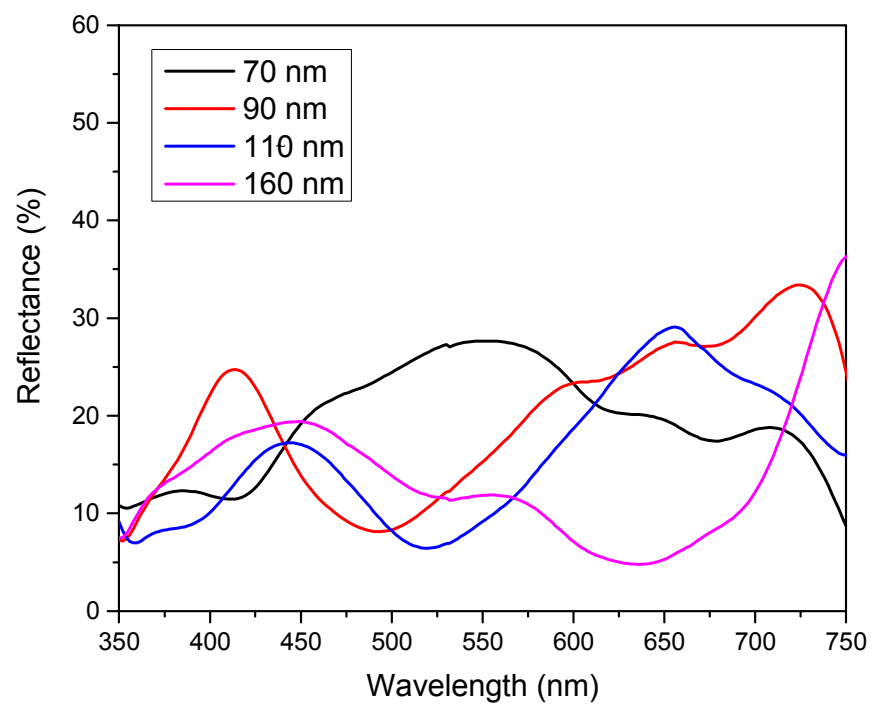

Figure S9. Reflectivity measurements of the tandem devcies with different perovskite layer thickness. The perovskite layer thickness shows significant influence on the reflectance of the real tandem devices with small (tens of nanometers) change in perovskite thickness, indicating a different optical distribution across the device.

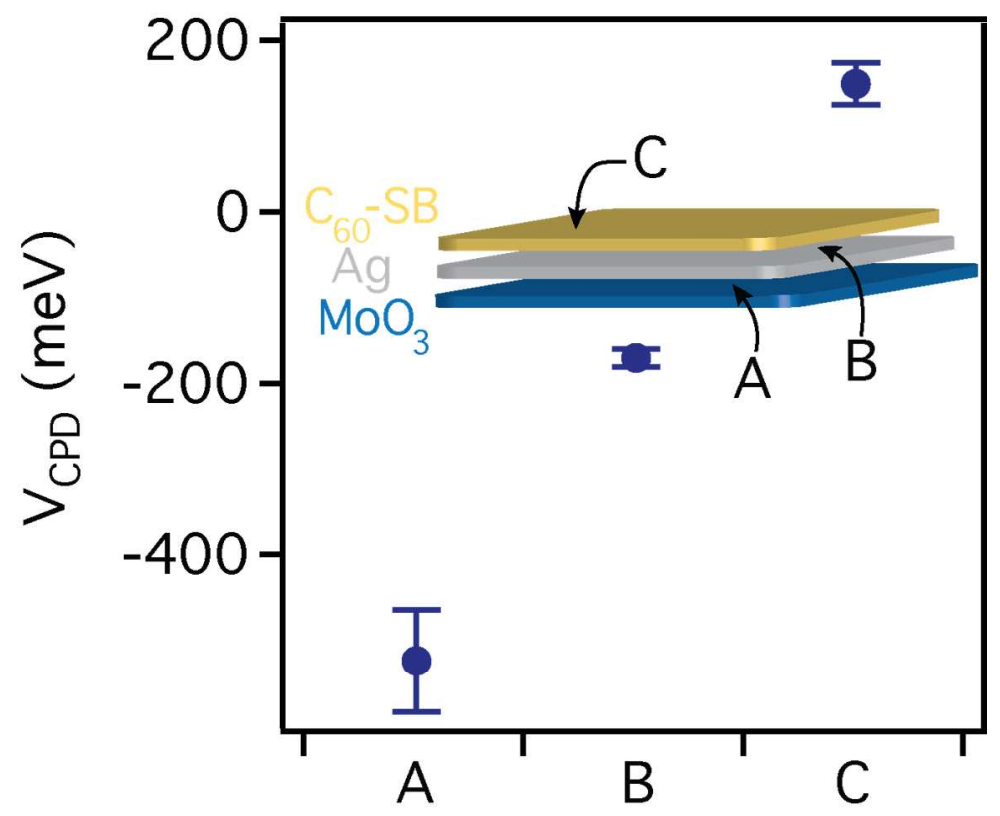

Figure S10. Surface potentials $\left(\mathrm{V}_{\mathrm{CPD}}\right)$ from $\mathrm{MoO}_{3}, \mathrm{Ag}$, to $\mathrm{C}_{60}-\mathrm{SB}$ of the graded recombination layer on gold substrates. The surface potential difference from $\mathrm{MoO}_{3}, \mathrm{Ag}$, to $\mathrm{C}_{60}-\mathrm{SB}$ (here) is even larger than that from $\mathrm{C}_{60} \mathrm{SB}, \mathrm{Ag}$, to $\mathrm{MoO}_{3}$ as shown in the main text, which further confirmed the graded potential property of the recombination layer. 